\title{
OBESIDADE EM IDOSOS ACOMPANHADOS PELA ESTRATÉGIA DE SAÚDE DA FAMÍLIA
}

\author{
Daiana Argenta Kümpel1, Adriana de Camargo Sodré2, Dalva Maria Pomatti³, Helenice de Moura \\ Scortegagna ${ }^{4}$, Josane Filippi ${ }^{5}$, Marilene Rodrigues Portella ${ }^{6}$, Marlene Doring ${ }^{7}$, Marina Scariot ${ }^{8}$
}

\footnotetext{
${ }^{1}$ Mestranda em Envelhecimento Humano pela Universidade de Passo Fundo (UPF). Nutricionista. Rio Grande do Sul, Brasil. E-mail: dkumpel84@hotmail.com

${ }^{2}$ Acadêmica do Curso de Enfermagem da UPF. Bolsista do PET-Saúde. Rio Grande do Sul, Brasil. E-mail: sodreadri@yahoo.com.br ${ }^{3}$ Mestre em Enfermagem. Professora do Curso de Enfermagem da UPF. Rio Grande do Sul, Brasil. E-mail: dalvamp@terra.com.br ${ }^{4}$ Doutora em Enfermagem. Professora do Curso de Enfermagem da UPF. Rio Grande do Sul, Brasil. E-mail:helenice@upf.br

${ }^{5}$ Acadêmica do Curso de Enfermagem da UPF. Bolsista do projeto de pesquisa PROCUIDAI e voluntária do PET-Saúde. Rio Grande do Sul, Brasil. E-mail: josanefilippi@hotmail.com

${ }^{6}$ Doutora em Enfermagem. Professora do Curso de Enfermagem da UPF. Rio Grande do Sul, Brasil. Tutora do PET-Saúde/ UPF. E-mail: portella@upf.br

${ }^{7}$ Doutora em Saúde Pública. Professora do Curso de Enfermagem da UPF. Rio Grande do Sul, Brasil. E-mail: doring@upf.br

${ }^{8}$ Acadêmica do Curso de Enfermagem da UPF. Rio Grande do Sul, Brasil. E-mail: marinascariot@hotmail
}

\begin{abstract}
RESUMO: A obesidade eleva as taxas de morbimortalidade, sendo um problema de saúde pública. Realizou-se estudo transversal com 123 participantes, objetivando identificar a proporção de casos e os fatores de risco para o sobrepeso/obesidade entre idosos com sessenta anos ou mais, atendidos por quatro equipes de Estratégia de Saúde da Família, de Passo Fundo-RS. Considerou-se variável dependente a obesidade e as demais como independentes: sexo, idade, renda familiar, escolaridade, ocupação, atividade física, tabagismo, medicação. As medidas antropométricas aferidas foram: peso, estatura, circunferência da cintura, quadril e cervical. Realizou-se análise bivariada usando o teste qui-quadrado de Pearson com um nível de significância $<0,05$. No modelo múltiplo permaneceram associadas à obesidade do idoso, a circunferência abdominal $(p=0,000)$ e a circunferência cervical $(p=0,000)$. No grupo estudado, sobrepeso/obesidade atingiu uma proporção de 49,6\%. Os resultados indicam alta prevalência de sobrepeso/obesidade, reforçando a necessidade de estratégias por parte dos profissionais de saúde, visando bem-estar e longevidade.
\end{abstract}

DESCRITORES: Obesidade. Saúde do idoso. Serviços de saúde.

\section{OBESITY AMONG ELDERLY ACCOMPANIED BY THE BRAZILIAN FAMILY HEALTH STRATEGY}

\begin{abstract}
Obesity increases morbidity and mortality rates and is a public health problem. A cross-sectional study involving 123 participants was carried out in order to identify the proportion of cases and risk factors among the overweight/obese elderly who receive care from four Family Health Strategy teams in the city of Passo Fundo, RS, Brazil. Obesity was considered the dependent variable and the remaining others as independent: gender, age, family income, education, occupation, physical activity, smoking, medication. The following anthropometric measurements were taken: weight, height, waist circumference, hip, and neck. We conducted bivariate analysis using the chi-square test with a $<0.05$ significance level. In the multiple model, the factors waist circumference $(p=0.000)$ and neck circumference $(p=0.000)$ remained associated with obesity among the elderly. In the group studied, excess weight / obesity has reached a proportion of $49.6 \%$. The results indicate the high prevalence of excess weight/obesity, reinforcing the need for strategies from health professionals who seek wellness and longevity
\end{abstract}

DESCRIPTORS: Obesity. Elderly's health. Health services.

\section{LA OBESIDAD EN ANCIANOS ACOMPAÑADOS POR EL PROGRAMA ESTRATEGIA DE SALUD FAMILIAR}

\begin{abstract}
RESUMEN: La obesidad aumenta las tasas de morbilidad y mortalidad y es un problema de salud pública. Se trata de un estudio transversal que se llevó a cabo con 123 participantes, con el objetivo de identificar la proporción de casos y los factores de riesgo para el sobrepeso/obesidad entre ancianos de sesenta años o más, asistidos por cuatro equipos del programa Estrategia de Salud Familiar, en la ciudad de Passo Fundo-RS. Se consideró a la obesidad como la variable dependiente y las demás como variables independientes: sexo, edad, ingresos familiares, educación, ocupación, actividad física, tabaquismo, medicamentos. Se consideraron como mediciones antropométricas: el peso, talla, circunferencia de la cintura. la cadera y el cuello. Se hizo un análisis bivariado mediante el test de Chi cuadrado con un nivel de significancia $<0,05$. En el grupo de estudio, el sobrepeso/obesidad ha alcanzado una proporción de $49,6 \%$. En el modelo de múltiples permanecieron asociadas a la obesidad de los ancianos, la circunferencia de la cintura $(p=0,000)$ y la circunferencia del cuello $(\mathrm{p}=0,000)$. Los resultados indican una alta prevalencia de sobrepeso/obesidad, lo que refuerza la necesidad de estrategias por parte de los profesionales de la salud que buscan el bienestar y la longevidad.
\end{abstract}

DESCRIPTORES: Obesidad. Salud del anciano. Servicios de salud. 


\section{INTRODUÇÃO}

A obesidade, excesso de tecido adiposo no organismo, tem sido considerada um sério problema de saúde pública, atingindo tanto países desenvolvidos como os em desenvolvimento. Considerada uma doença integrante do grupo de Doenças Crônicas Não- Transmissíveis (DCNTs), as morbimortalidades relacionadas são doenças cardiovasculares, osteomusculares, neoplásicas, as quais favorecem o aparecimento de enfermidades, influenciando direta ou indiretamente na saúde do indivíduo. ${ }^{1-2}$

As DCNTs têm história natural prolongada, interação de fatores etiológicos desconhecidos, manifestações clínicas com períodos de remissão e de exacerbação, podendo evoluir para diferentes graus. A industrialização e as mudanças nos padrões de vida e trabalho são fatores que contribuem para transformação nos padrões das doenças não transmissíveis. Essas transformações apresentam maior impacto nos países em desenvolvimento..$^{3-4}$ No que tange à obesidade, podemos citar fatores específicos, tais como: ambientais (falta de tempo para praticar atividades físicas, dieta hipercalórica); genéticos e de história familiar; saúde (problemas hormonais, ansiedade, depressão) e idade (diminuição da massa corporal e do gasto energético). ${ }^{1,5}$

No Brasil, observamos índices elevados de obesidade em populações de baixa renda, especialmente em mulheres, em razão do seu maior número e longevidade em relação aos homens. Outro aspecto relevante é que elas procuram mais atendimento à saúde do que o sexo oposto. Esse fato se reflete na maior taxa de mulheres em grupos etários mais velhos, correspondendo a aproximadamente dois terços da população acima de 75 anos, em países como Brasil e África do Sul. ${ }^{1-3,5-6}$

Com o envelhecimento humano ocorrem alterações corporais, como a redução da altura, acentuada cifose dorsal, redução da massa corporal magra, acúmulo da gordura visceral e diminuição da quantidade de água no organismo. Ainda não são bem conhecidas as consequências dessas alterações, porém sabe-se que há diferença no risco de doenças cardiovasculares associadas à redistribuição de gordura, que é diferente nos dois sexos. Por conta disso, ocorrem modificações em alguns indicadores de gordura e de massa muscular, os quais são comumente avaliados em estudos antropométricos em idosos. ${ }^{2,7-9}$
O Índice de Massa Corporal (IMC), como indicador de adiposidade no idoso, tem sido considerado pobre em razão de não refletir a redistribuição regional de gordura, porém é bastante utilizado em virtude de sua fácil aplicação e baixo custo, o qual se adequa à realidade brasileira, assim justificando seu uso em estudos epidemiológicos. ${ }^{6-7,10}$

Por sua vez, ressalta-se que algumas doenças comumente são potencializadas pela obesidade, assumindo maior importância entre os indivíduos idosos, haja vista que este segmento populacional já apresenta índices de enfermidades aumentados com a idade, mesmo naqueles não obesos. ${ }^{2}$

Assim, a morbimortalidade provocada pela obesidade associada ao processo do envelhecimento fisiológico e o quadro de transição demográfica e epidemiológica apontam para a necessidade de atenção à obesidade, integrando políticas econômicas e de saúde dentro do processo de adoecer e morrer do segmento populacional sob cobertura da Estratégia de Saúde da Família (ESF). Para isso exige-se a educação permanente dos profissionais de saúde envolvidos com a atenção básica, qualificando a assistência de acordo com os princípios do Sistema Único de Saúde.

O objetivo deste estudo foi identificar a proporção de casos e os fatores de risco de sobrepeso/obesidade entre idosos atendidos em ESFs na cidade de Passo Fundo-RS.

\section{METODOLOGIA}

Trata-se de estudo transversal com idosos atendidos pela ESF, no período de março a novembro de 2009, no município de Passo Fundo, localizado no norte do Estado do Rio Grande do Sul, Brasil.

A amostra foi constituída por 123 idosos com idade igual ou superior a sessenta anos, de ambos os sexos, atendidos na primeira consulta de enfermagem, em quatro unidades de ESF que recebiam acadêmicos do Curso de Enfermagem da Universidade de Passo Fundo, para desenvolvimento da Disciplina Estágio Curricular. Os dados foram obtidos utilizando-se um questionário estruturado, como instrumento para entrevista com os idosos, durante a consulta de enfermagem realizada pelos acadêmicos do curso, conforme a demanda espontânea da clientela.

Excluíram-se aqueles com alterações cognitivas, conforme indicação do teste rápido, da avaliação multidimensional rápida da pessoa 
idosa, sugerido pelo Ministério da Saúde ${ }^{11}$ e os incapazes de permanecer em pé para pesagem e determinação das medidas antropométricas.

Consideraram-se como variável dependente a obesidade; independentes, as variáveis sóciodemográficas: sexo, idade, renda familiar mensal (renda total, em salário mínimo, dos integrantes da família), escolaridade (analfabeto, número de anos cursados de um a quatro anos, de cinco a oito anos e nove anos ou mais) e ocupação autorreferida no momento da entrevista; além das variáveis comportamentais: prática de atividade física regular quanto à frequência (menos de três vezes por semana, três vezes ou mais) e duração (menos de 30 minutos, 30 minutos ou mais); tabagismo (fumantes, ex-fumantes, pessoas que nunca fumaram); uso de medicação para diabetes melitus; e uso regular de medicamentos anti-hipertensivos.

Ainda, como variáveis independentes consideraram-se as medidas para determinação do diagnóstico do estado nutricional: peso, estatura, circunferência da cintura, quadril e circunferência cervical. A aferição antropométrica seguiu normas padronizadas. ${ }^{12} \mathrm{O}$ peso foi medido em quilogramas, utilizando-se balança de plataforma mecânica com antropômetro acoplado (Filizzola ${ }^{\circledR}$ ), com capacidade de até $150 \mathrm{~kg}$ e precisão de 100 gramas, previamente regulada. Os idosos foram pesados trajando roupas leves e sem sapatos. A estatura foi medida em metros, com os idosos descalços em cima da plataforma da balança, de costas para seu marcador, com os pés unidos, em posição ereta e olhar fixo na altura da linha do horizonte, então, a vareta horizontal do antropômetro é baixada até o vértex da cabeça, pressionando o cabelo.

A obesidade e o sobrepeso foram avaliados mediante o cálculo do IMC, pela divisão do valor do peso (em quilogramas) pela estatura (em metros quadrados), sendo codificadas utilizando-se os pontos de corte para idosos: igual ou menor de 22 $\mathrm{Kg} / \mathrm{m}^{2}$ - baixo peso; mais de 22 e menos de $27 \mathrm{~kg} /$ $\mathrm{m}^{2}$ - adequado ou eutrófico; igual ou maior de 27 $\mathrm{Kg} / \mathrm{m}^{2}$ - sobrepeso. ${ }^{11}$

As circunferências abdominal e quadril foram mensuradas utilizando-se fita métrica inelástica. A Circunferência Abdominal (CA) foi obtida durante a expiração normal, sendo circundada a menor circunferência horizontal no ponto médio entre a última costela e a crista ilíaca. Aqueles com CA acima de $102 \mathrm{~cm}$, no caso de homens, e acima de $88 \mathrm{~cm}$, em se tratando de mulheres, foram caracterizados como portadores de obesidade abdominal. ${ }^{12}$
A Circunferência do Quadril (CQ) foi verificada sobre a região glútea, sendo circundada a maior circunferência horizontal. A relação da cintura para o quadril (RCQ) é calculada dividindo-se a medida da circunferência da cintura $(\mathrm{cm})$ pela do quadril $(\mathrm{cm})$. É fortemente associada à gordura visceral, sendo um índice aceitável de gordura intra-abdominal. É a medida de adiposidade mais frequentemente utilizada. Foram utilizados para Relação Cintura/Quadril (RCQ) os pontos de corte: 1,0 ou mais para homens e 0,8 ou mais para mulheres. ${ }^{12}$

A Circunferência Cervical (CC) foi verificada com fita métrica colocada na altura da cartilagem cricotireóidea, mensurada em centímetros. Valores normais em homens são $43 \mathrm{~cm}$ e em mulheres, $38 \mathrm{~cm} \cdot{ }^{13}$

A medida da pressão arterial foi realizada no braço direito, com o idoso sentado, após cinco minutos de repouso, com o braço direito exposto. Os valores admitidos são: 120x80 mmHg, em que a pressão arterial é considerada ótima e 130x85 $\mathrm{mmHg}$, considerada limítrofe. Valores pressóricos superiores a 140x90 mmHg denotam Hipertensão Arterial Sistêmica (HAS). ${ }^{14}$

As análises estatísticas foram realizadas com auxílio do software Stata versão 10. Para verificar as associações entre variáveis categóricas dependente e independentes utilizou-se o teste qui-quadrado de Pearson ou exato de Fisher, a um nível de significância menor que 0,05. Para calcular a razão de chances (odds ratio) foi utilizada análise de regressão logística múltipla. Variáveis com $p £ 0,20$ foram selecionadas para inclusão na análise. No modelo final foram mantidas as variáveis estatisticamente associadas com a variável dependente $(\mathrm{p}<0,05)$.

Este estudo seguiu a Resolução 196/96 do Conselho Nacional de Saúde para pesquisa envolvendo seres humanos, incluindo a garantia de sigilo das informações, privacidade, seu consentimento livre e esclarecido, entre outros direitos. Teve aprovação pelo Comitê de Ética em Pesquisa da UPF, protocolo CEP 303/2008, CAAE n.0104.0.398.000-08.

\section{RESULTADOS E DISCUSSÃO}

Dos 123 idosos avaliados, 86,9\% eram do sexo feminino. Alguns fatores podem explicar essa situação, tais como: a maior longevidade feminina, quando confrontada com homens em grupos etários mais velhos, sendo na população brasileira 
essa diferença por sexo de oito anos; maior solidão das mulheres em relação aos homens; menor exposição feminina a determinados fatores de risco e maior preocupação com o estado de saúde, enfatizada pela maior assistência gineco-obstétrica, que faz com que as mulheres procurem mais a Unidade de Saúde, conforme é demonstrado em diversos estudos. ${ }^{12,15-19}$

Quanto à escolaridade, houve predomínio de cinco a oito anos de estudo $(51,6 \%)$ em ambos os sexos, o que difere de outros estudos, nos quais o analfabetismo foi mais prevalente na população masculina com cinquenta anos ou mais. ${ }^{20}$

A maioria dos idosos é aposentada (60,2\%), com renda familiar mensal de até três salários mínimos $(67,8 \%)$. Referentemente à renda familiar, a insuficiência financeira da maioria da população idosa, dependente de aposentadorias, muitas vezes comprometida com a aquisição de medicamentos, determina como prioridade a compra de alimentos de baixo custo, de pouca qualidade nutricional e com maior aporte calórico. ${ }^{21}$

No entanto, as variáveis socioeconômicas podem ser influenciadas pela obesidade, ou seja, a obesidade pode precedê-las, mencionando-se que pessoas com excesso de peso têm renda mais baixa e completaram menos anos na escola. ${ }^{22}$

A prática da atividade física foi relatada por $81,3 \%$ dos idosos, dos quais, 53\% praticavam-na menos de três vezes por semana e $47 \%$, três vezes ou mais. Ainda, a maioria (98\%) relatou praticar atividade física com duração de 30 minutos ou mais, o que contribui para o bem-estar, sendo benéfica, inclusive, para portadores de doenças crônicas, mesmo quando a prática de atividade física é iniciada em uma fase tardia da vida por sujeitos sedentários. ${ }^{23}$

Quanto ao tabagismo, a maioria $(84,9 \%)$ referiu não ser fumante. Cabe ressaltar que alguns estudos constataram maior prevalência de tabagistas entre indivíduos do sexo masculino, ${ }^{24-25}$ fato que justifica o baixo percentual de fumantes no presente estudo, em razão da maior prevalência do sexo feminino.

No que se refere ao uso regular de medicamentos, 18,7\% dos idosos utilizam medicação para diabetes melitus, enquanto que $70,7 \%$ fazem uso de medicamentos anti-hipertensivos. Em revisão de oito ensaios incluindo mais de 15.000 pacientes com sessenta anos ou mais, observou-se que o uso de medicação anti-hipertensiva reduz em 13\% a mortalidade, em $30 \%$ o AVC e em $23 \%$ os eventos coronarianos. ${ }^{26}$
$\mathrm{Na}$ população estudada verificou-se que a maioria (49,6\%) apresentou sobrepeso/obesidade, $45,5 \%$ dos idosos estão com o peso adequado e $4,9 \%$ apresentam baixo peso. O resultado quanto ao sobrepeso é preocupante, principalmente pela associação da obesidade com várias doenças crônicas não transmissíveis, que acarretam aumento da morbi-mortalidade e impacto sobre o sistema de saúde, além de diminuir a qualidade de vida dos idosos. ${ }^{27}$

Em estudo relativo à Pesquisa Nacional sobre Saúde e Nutrição, de 1989, aproximadamente metade $(50,2 \%)$ das mulheres idosas avaliadas apresentou sobrepeso, enquanto entre os homens idosos a prevalência foi de $30,4 \%{ }^{28}$

$\mathrm{Na}$ análise bivariada não houve associação estatisticamente significativa entre obesidade e sexo, faixa etária, RCQ e tabagismo ( $p>0,05)$. Houve associação entre obesidade e circunferência abdominal, circunferência cervical e HAS $(p<0,05)$ (Tabela 1).

Tabela 1 - Características dos idosos acompanhados pela Estratégia de Saúde da Família, Passo Fundo-RS, 2009

\begin{tabular}{|c|c|c|c|c|c|c|}
\hline \multirow{3}{*}{ Variáveis } & \multicolumn{4}{|c|}{ Obesidade } & \multirow{3}{*}{ OR* bruto $\left(\mathrm{IC}_{95 \%}\right)$} & \multirow{3}{*}{$\mathbf{p}$} \\
\hline & \multicolumn{2}{|c|}{ Sim } & \multicolumn{2}{|c|}{ Não } & & \\
\hline & $\mathbf{n}$ & $\%$ & $\mathbf{n}$ & $\%$ & & \\
\hline \multicolumn{7}{|l|}{ Sexo } \\
\hline Feminino & 53 & 86,9 & 54 & 87,0 & 1 & \\
\hline Masculino & 8 & 13,1 & 8 & 13,0 & $1,02(0,35-2,93)$ & 0,972 \\
\hline \multicolumn{7}{|l|}{ Faixa etária } \\
\hline $60-69$ & 30 & 49,2 & 31 & 50,0 & 1 & \\
\hline $70-79$ & 25 & 41,0 & 24 & 38,7 & $1,08(0,51-2,29)$ & 0,848 \\
\hline $80 \mathrm{e}+$ & 6 & 9,8 & 7 & 11,3 & $0,88(0,26-2,97)$ & 0,844 \\
\hline \multicolumn{7}{|c|}{ Circunferência cervical } \\
\hline Normal & 39 & 63,9 & 58 & 95,1 & 1 & \\
\hline Risco & 22 & 36,1 & 3 & 4,9 & $10,9(2,73-43,6)$ & 0,000 \\
\hline \multicolumn{7}{|c|}{ Circunferência abdominal } \\
\hline Normal & 9 & 14,8 & 37 & 59,7 & 1 & \\
\hline Risco & 52 & 85,2 & 25 & 40,3 & $8,5(3,19-22,95)$ & 0,000 \\
\hline \multicolumn{7}{|c|}{ Razão cintura/quadril } \\
\hline Normal & 6 & 9,8 & 10 & 16,0 & 1 & \\
\hline Risco & 55 & 90,2 & 52 & 84,0 & $1,76(0,59-5,24)$ & 0,300 \\
\hline \multicolumn{7}{|l|}{ Has } \\
\hline Sim & 54 & 88,5 & 46 & 74,2 & 1 & \\
\hline Não & 7 & 11,5 & 16 & 25,8 & $0,37(0,14-1,01)$ & 0,042 \\
\hline \multicolumn{7}{|c|}{ Atividade física } \\
\hline Sim & 47 & 78,3 & 53 & 85,5 & 1 & \\
\hline Não & 13 & 21,7 & 9 & 14,5 & $1,63(0,63-4,19)$ & 0,306 \\
\hline
\end{tabular}


No modelo múltiplo, a HAS perdeu a significância, permanecendo associada à obesidade do idoso, à circunferência abdominal e à circunferência cervical $(p=0,000)$. A chance de uma pessoa com circunferência abdominal alterada apresentar obesidade é 6,61 vezes se comparada com uma pessoa que não apresenta essa característica, a qual prevaleceu alterada em $67,5 \%$ dos idosos deste estudo. Da mesma forma, pode-se observar que a chance de uma pessoa com circunferência cervical alterada ser obesa é 6,68 vezes maior que uma pessoa com valores normais, sendo prevalente em $88,0 \%$ dos idosos, esse quadro irá interferir na qualidade do sono, aumentando a possibilidade de acidentes e interferindo no rendimento do trabalho. ${ }^{29}$ (Tabela 2).

Tabela 2 - Características dos idosos e fatores associados à obesidade em idosos acompanhados pela Estratégia de Saúde da Família, Passo Fundo-RS, 2009

\begin{tabular}{|c|c|c|c|c|c|c|}
\hline \multirow{2}{*}{ Variáveis } & \multirow{2}{*}{ Total } & \multicolumn{2}{|c|}{ Obesidade } & \multirow{2}{*}{$\mathrm{OR}_{\text {ajustada }}$} & \multirow{2}{*}{ IC95\% } & \multirow[b]{2}{*}{$\mathbf{p}$} \\
\hline & & $\mathbf{n}$ & $\%$ & & & \\
\hline \multicolumn{7}{|c|}{ Circunferência abdominal } \\
\hline Normal & 46 & 9 & 19,6 & 1 & & \\
\hline Risco & 77 & 52 & 67,5 & 6,61 & $2,67-16,38$ & 0,000 \\
\hline \multicolumn{7}{|c|}{ Circunferência cervical } \\
\hline Normal & 97 & 39 & 40,2 & 1 & & \\
\hline Risco & 25 & 22 & 88,0 & 6,68 & $1,76-25,33$ & 0,005 \\
\hline
\end{tabular}

Recentemente, estudos demonstraram a gravidade de se ter a circunferência abdominal aumentada, porque representa risco para o desenvolvimento de doenças crônicas não transmissíveis, dentre as quais doenças cardiovasculares. ${ }^{30,31}$

Por sua vez, a medida da circunferência cervical dentro dos padrões de normalidade significa que as pessoas apresentam baixo risco de serem portadoras da síndrome da apneia obstrutiva do sono. ${ }^{13}$

Para que os idosos, em especial os predispostos a sobrepeso e obesidade, possam se beneficiar das práticas de promoção à saúde desenvolvidas pelos enfermeiros da ESF é necessário que se invista em iniciativas na perspectiva de um atendimento integral à saúde do idoso. Vale destacar que a concretização das mudanças vai além das possibilidades dos profissionais das ESFs. Todavia, a atual conformação da equipe básica da ESF não é suficiente para atender às necessidades desse segmento populacional, denotando a urgência da agregação à equipe, entre outros profissionais, de educador físico e nutricionista. ${ }^{32}$

\section{CONCLUSÃO}

Este estudo apontou alta prevalência de sobrepeso/obesidade no grupo de idosos e os fatores agravantes que se associaram foram a circunferência abdominal e a circunferência cervical. Diante da importância da composição corporal sobre a saúde do idoso, percebe-se a necessidade de estudos mais abrangentes, que possam arrolar uma amostra representativa atendida pela ESF da cidade de Passo Fundo, para demonstrar a prevalência da obesidade na população idosa neste município.

Os resultados obtidos contribuem para a reflexão acerca da concretização das propostas das políticas de saúde para atenção aos idosos, atendendo aos diferentes contextos. Igualmente, alerta-se para a necessidade do enfermeiro da ESF reorganizar seu processo de trabalho, assumindo um dimensionamento ampliado na proposição de ações de saúde que articulem seus saberes com os demais profissionais. As questões da obesidade e do envelhecimento humano requerem atenção interdisciplinar.

Por outro lado, é pertinente que se considere a questão da educação permanente, em especial para os profissionais das ESF, com vistas ao planejamento e à proposição de ações efetivas alinhadas à consolidação do Sistema Único de Saúde, construindo uma atenção básica eficiente e resolutiva para os diferentes segmentos etários.

\section{REFERÊNCIAS}

1. Pinheiro ARO, Freitas SFT, Corso ACT. Uma abordagem epidemiológica da obesidade. Rev Nutr [online]. 2004 [acesso 2010 Mar 30]; 17(4):52333. Disponível em: http://www.scielo.br/scielo. php?pid=S1415-52732004000400012\&script $=$ sci arttext 
2. Cabrera MAS, Jacob Filho W. Obesidade em idosos: prevalência, distribuição e associação com hábitos e co-morbidades. Arq Bras Endocrinol Metab [online]. 2001 [acesso 2010 Mar 30]; 45(5):494-501. Disponível em: http://www.scielo.br/scielo.php?script=sci_ar ttext\&pid=S0004-27302001000500014

3. World Health Organization. Envelhecimento ativo: uma política de saúde. Brasília (DF): Organização Pan-Americana da Saúde; 2005.

4. Pontes LM, Sousa MSC, Silva JMFL, Gomes ERM, Reis EES, Lira FAS. Análise da qualidade de vida e prevalência de sobrepeso em moradores da zona rural do município de Pombal. Rev Saúde Com [online]. 2005 [acesso 2010 Mar 30]; 1(1):18-23. Disponível em: http://www.uesb.br/revista/rsc/ v1/v1n1a3.pdf

5. Banco de Saúde. Causas da obesidade. [página da internet]. [acesso 2010 Mar 30]. Disponível em: http://www.bancodesaude.com.br/obesidade/ causas-obesidade

6. Buzzachera CF, Krause MP, Elsangedy HM, Hallage T, Granato P, Krinski K, Campos W, Silva SG. Prevalência de sobrepeso e obesidade geral e central em mulheres idosas da cidade de Curitiba, Paraná. Rev Nutr [online]. 2008 [acesso 2010 Mar 28]; 21(5):525-33. Disponível em: http:/ / www.scielo.br/ scielo.php?script $=$ sci_arttext\&pid $=S 1415527320080$ 00500005\&lng $=$ pt\&nrm $=$ iso

7. Sampaio LR, Figueiredo, VC. Correlação entre o índice de massa corporal e os indicadores antropométricos de distribuição de gordura corporal em adultos e idosos. Rev Nutr [online]. 2005 [acesso 2010 Mar 10]; 18(1):53-61. Disponível em: http:/ / www.scielo.br/scielo.php?pid=S141552732005000100005\&script=sci_arttext

8. Menezes TN, Marucci MFN. Perfil dos indicadores de gordura e massa muscular corporal dos idosos de Fortaleza, Ceará, Brasil. Cad Saúde Pública [online]. 2007 [acesso 2010 Mar 17]; 23(12):2887-95. Disponível em: http://www.scielo.br/scielo.php?script=sci arttext\&pid=S0102-311X2007001200010

9. Santos DM, Sichieri R. Índice de massa corporal e indicadores antropométricos de adiposidade em idosos. Rev Saúde Pública [online]. 2005 [acesso 2010 Mar 17]; 39(2):163-8. Disponível em: http:/ / www.scielosp.org/ scielo.php?pid=S003489102005000200004\&script=sci_arttext

10. Garcia ANM, Romani SAM, Lira PIC. Indicadores antropométricos na avaliação nutricional de idosos: um estudo comparativo. Rev Nutr [online]. 2007[acesso 2010 Mar 30]; 20(4):371-8. Disponível em: http://www.scielo.br/scielo.php?script=sci_ar ttext\&pid=S1415-52732007000400004

11. Ministério da Saúde (BR). Envelhecimento e saúde da pessoa idosa: cadernos de atenção básica, n. 19. Brasília (DF): Ministério da Saúde; 2006.
12. World Health Organization. Defining the problem of overweight and obesity. In: World Health Organization. Obesity: preventing and managing the global epidemic: report of a Who Consultation (WHO Technical Report Series, 894). Geneva: WHO; 2000. p. 241-3.

13. Soares MC. Síndrome da apnéia e hipopnéia obstrutiva do sono (SAHOS). São Paulo: UNIFESP, 2005.

14. Organização Mundial de Saúde. Consenso brasileiro de hipertensão arterial 3. Campos do Jordão (SP): OMS; 1998.

15. Santos MRDR, Mendes SCSM, Morais DB, Coimbra MPSM, Araújo MAM, Carvalho CMRG. Caracterização nutricional de idosos com hipertensão arterial em Teresina, PI. Rev Bras Geriatr Gerontol. 2007; 10(1):73-86.

16. Tinoco ALA, Brito LF, Sant'Anna MSL, Abreu WC, Mello AC, Silva MMS, et al. Sobrepeso e obesidade medidos pelo índice de massa corporal (IMC), circunferência da cintura (CC) e relação cintura/ quadril (RCQ), de idosos de um município da Zona da Mata Mineira. Rev Bras Geriatr Gerontol. 2006; 9(2):63-73.

17. Pinheiro RS, Viacava F, Travassos C, Brito AS. Gênero, morbidade, acesso e utilização de serviços de saúde no Brasil. Ciênc Saúde coletiva [online]. 2002 [acesso 2010 Mar 17]; 7(4):687-707. Disponível em: http:/ / www.scielo.br/scielo.php?pid=S1413$81232002000400007 \&$ script=sci_arttext

18. Moreira AJ, Nicastro H, Cordeiro RC, Coimbra P, Frangella VS. Composição corporal de idosos segundo a antropometria. Rev Bras Geriatr Gerontol [online]. 2009 [acesso 2010 Mar 19]; 12(2):20113. Disponível em: http://revista.unati.uerj. br / scielo.php?script=sci_abstract\&pid=S180998232009000200005\&lng=pt\&nrm=iso

19. Afio CJ, Costa AC, Santos ZMSA, Soares E. Descrição dos fatores de risco para alterações cardiovasculares em um grupo de idosos. Texto Contexto Enferm [online]. 2008 [acesso 2010 Mar 19]; 17(2):327-35. Disponível em: http://www.scielo.br/scielo. php?pid=S0104-07072008000200015\&script $=$ sci abstract\&tlng $=p t$

20. Florindo AA, Latorre MRDO, Tanaka T, Jaime $\mathrm{PC}$, Zerbini CAF. Fatores associados à prática de exercícios físicos em homens voluntários adultos e idosos residentes na Grande São Paulo, Brasil. Rev Bras Epidemiol [online]. 2001 [acesso 2010 Mar 17]; 4(2):105-13. Disponível em: http://www.scielosp. org/scielo.php?script=sci_arttext\&lng=pt\&nrm=is o\&tlng=pt\&pid=S1415-790X2001000200005

21. Marques APO, Arruda IKG, Leal, MCC, Espírito SACG. Envelhecimento, obesidade e consumo alimentar em idosos. Rev Bras Geriatr Gerontol. 2007; 10(2):231-42. 
22. Gigante DP, Barros FC, Post CLA, Olinto MTA Prevalência de obesidade em adultos e seus fatores de risco. Rev Saúde Pública [online]. 1997 [acesso 2010 Mar 19]; 31(3):236-46. Disponível em: http:/ / www.scielosp.org/ scielo.php?pid=S003489101997000300004\&script=sci_arttext

23. Caromano, FA; Ide, MR; Kerbauy, RR. Manutenção na prática de exercícios por idosos. Rev Dep Psicol UFF [online]. 2006 [acesso 2010 Mar 30]; 18(2):177-92. Disponível em: http://www. scielo.br/scielo.php?script=sci_arttext\&pid $=$ S0104-80232006000200013

24. Luppi CHB, Alves MVFF, Santos AA. Programa de cessação ao tabagismo: perfil e resultados. Rev Ciênc Ext [online]. 2006 [acesso 2010 Mar 19]; 2(2):62. Disponível em: http://ojs.unesp.br/index.php/ revista_proex/article/viewArticle/196

25. Ministério da Saúde (BR). Instituto Nacional de Câncer. Tabagismo: dados e números. Rio de Janeiro (RJ): INCA; 2004.

26. Jobim, EFC. Hipertensão arterial no idoso: classificação e peculiaridades. Rev Soc Bras Clín Méd. 2008 nov.-dez; 6(6):250-3.

27. Silveira EA, Kac G, Barbosa LS. Prevalência e fatores associados à obesidade em idosos residentes em Pelotas, Rio Grande do Sul, Brasil: classificação da obesidade segundo dois pontos de corte do índice de massa corporal. Cad Saúde Pública [online]. [acesso 2010 Mar 26] 2009; 25(7):1569-77. Disponível em: http:/ / www.scielo.br/scielo.php?pid=S0102311X2009000700015\&script=sci_abstract\&tlng=pt

28. Tavares EL, Anjos LA. Perfil antropométrico da população idosa brasileira. Resultados da Pesquisa Nacional sobre Saúde e Nutrição. Cad Saúde Pública. 1999; 15(4):759-68.

29. Segal A, Mansini M. Tudo que você precisa saber antes de reduzir seu estômago - Guia completo da cirurgia da obesidade. São Paulo (SP): Brasiliense, 2007.

30. Girotto, E; Andrade, SM; Cabrera, MAS. Prevalência de obesidade abdominal em hipertensos cadastrados em uma Unidade de Saúde da Família. Arq Bras Cardiol. 2010 Jun; 94(6):754-62.

31. Sposito AC, Caramelli B, Fonseca FAH, Bertolami MC, Afiune Neto A, Souza AD, et AL. Sociedade Brasileira de Cardiologia: IV Diretriz brasileira sobre dislipidemias e prevenção da aterosclerose: Departamento de Aterosclerose da Sociedade Brasileira de Cardiologia. Arq Bras Cardiol. 2007; 88(supl.1):2-19.

32. Marin MJS, Martins AP, Marques F, Martins F, Feres $\mathrm{BOM}$, Saraiva AKH, Druzian S. A atenção à saúde do idoso: ações e perspectivas dos profissionais. Rev Bras Geriatr Gerontol. 2008; 11(2):245-58.
Correspondência: Daiana Argenta Kümpel Rua Sete de Setembro, 467, ap 802. Edifício Quebec 99010-121. Passo-Fundo, RS, Brasil

E-mail: dkumpel84@hotmail.com
Recebido: 20 de outubro de 2010 Aprovação: 08 de julho de 2011 